\title{
Reply to Letter to the Editor (JNC-12-151-LE) regarding "PET: Is myocardial flow quantification a clinical reality?"
}

We appreciate the letter from Johnson and Gould providing further information on their analysis software that enables quantification of myocardial blood flow based on retention kinetics and simplified acquisition protocol as compared with methods using compartmental modeling. The limitation of that software is that it currently allows analysis of data produced only by scanners manufactured by a single vendor. In addition, although the users have been apparently positive in their comments, rigorous direct comparison of this software against the others would be needed to judge, which method or software is better than the other.

We very much appreciate that the authors have earlier presented an algorithm for interpreting absolute flow and flow reserve data in clinical decision making. ${ }^{1}$ Such integrative algorithms are necessary for trials of revascularization guided by absolute flow measurements. Meanwhile, more studies have shown that noninvasive quantitative assessment of coronary vasodilator function with positron emission tomography is a powerful, independent predictor of cardiac mortality. Data from a large patient cohort of 2,783 patients with known or suspected coronary artery disease demonstrated the incremental prognostic value of flow reserve over semi-quantitative measures of myocardial ischemia and scar as well as other clinical variables for identification of patients at risk of cardiac death. ${ }^{2}$ The addition of flow reserve resulted in the correct reclassification of approximately one-third of all intermediate-risk patients. Another study provided evidence that among diabetic patients without coronary artery disease, those with impaired flow reserve had event rates comparable to patients with prior coronary artery disease while those with preserved flow reserve had event rates comparable to non-diabetics. ${ }^{3}$

\section{Acknowledgments}

The authors acknowledge financial support from The Academy of Finland Centre of Excellence in Molecular Imaging in Cardiovascular and Metabolic Research, Helsinki, Finland and Finnish Foundation for Cardiovascular Research

\section{Conflict of interest}

The authors have indicated that they have no financial conflicts of interest.

Antti Saraste, $M D, P h D^{a, b}$

e-mail:antti.saraste@utu.fi Sami Kajander, $M D, P h D^{a}$

Chunlei Han, MD, $P h D^{a}$

Sergey V. Nesterov, MD, PhD, PMP ${ }^{a}$

Juhani Knuuti, $M D, P h D^{a}$

e-mail:juhani.knuuti@utu.fi

${ }^{a}$ Turku PET Centre, University of Turku, Kiinamyllynkatu 4-8, 20520 Turku, Finland

${ }^{b}$ Department of Medicine, Turku University Hospital, Kiinamyllynkatu 4-8, 20520 Turku, Finland

\section{References}

1. Johnson NP, Gould KL. Integrating noninvasive absolute flow, coronary flow reserve, and ischemic thresholds into a comprehensive map of physiological severity. JACC Cardiovasc Imaging 2012;5:430-40

2. Murthy VL, Naya M, Foster CR, Hainer J, Gaber M, Di Carli G, et al. Improved cardiac risk assessment with noninvasive measures of coronary flow reserve. Circulation 2011;124:2215-24.

3. Murthy VL, Naya M, Foster CR, Gaber M, Hainer J, Klein J, et al. Association between coronary vascular dysfunction and cardiac mortality in patients with and without diabetes mellitus. Circulation 2012. doi:10.1161/CIRCULATIONAHA.112.120402.

doi:10.1007/s12350-012-9629-3

J Nucl Cardiol 2012;19:1245.

$1071-3581 / \$ 34.00$

Copyright (C) 2012 American Society of Nuclear Cardiology. 